(1)

CrossMark

\title{
Towards tackling tuberculosis in vulnerable groups in the European Union: the E-DETECT TB consortium
}

\author{
Ibrahim Abubakar ${ }^{1}$, Alberto Matteelli², Gerard de Vries ${ }^{3}$, Dominik Zenner ${ }^{4}$, \\ Daniela M. Cirillo ${ }^{5}$, Knut Lönnroth ${ }^{6}$, Gilda Popescu ${ }^{7}$, Lucia Barcellini ${ }^{8}$, \\ Alistair Story ${ }^{9}$ and Giovanni Battista Migliori (ib ${ }^{10}$
}

\begin{abstract}
Affiliations: ${ }^{1}$ Institute for Global Health, University College London, London, UK. ${ }^{2}$ Institute of Infectious and Tropical Diseases, Infectious Disease, University of Brescia, Brescia, Italy. ${ }^{3}$ KNCV Tuberculosis Foundation, Netherlands and Elimination. The Hague, The Netherlands. ${ }^{4}$ Public Health England, National Infections Service, London, UK and University College London, London, UK. ${ }^{5}$ IRCCS San Raffaele Scientific Institute, Milan, Italy. ${ }^{6}$ Karolinska Institutet, Dept of Public Health Sciences, Stockholm, Sweden. ${ }^{7}$ Institutul de Pneumoftiziologie Marius Nasta, Bucuresti, Romania. ${ }^{8}$ IRCCS San Raffaele Scientific Institute (ORS), Milan, Italy. ${ }^{9}$ University College London Hospitals NHS Foundation Trust, Find and Treat, London, UK. ${ }^{10}$ WHO Collaborating Centre for TB and Lung Diseases, Maugeri Care and Research Institute, IRCCS, Tradate, Italy.
\end{abstract}

Correspondence: Ibrahim Abubakar, Institute for Global Health, University College London, London, UK. E-mail: i.abubakarducl.ac.uk

@ERSpublications

Tackling tuberculosis requires early diagnosis, treatment and innovative transdisciplinary action through pan-European projects http://ow.ly/1sf]30jy3VB

Cite this article as: Abubakar I, Matteelli A, de Vries G, et al. Towards tackling tuberculosis in vulnerable groups in the European Union: the E-DETECT TB consortium. Eur Respir J 2018; 51: 1702604 [https://doi. org/10.1183/13993003.02604-2017].

Tuberculosis (TB) in the European Union (EU) is increasingly a public health problem that disproportionately and increasingly affects risk groups. The 30 EU/European Economic Area (EEA) countries reported 60195 cases of TB in 2015; 4.1\% of which was multidrug-resistant (MDR) TB [1]. The European Respiratory Society, the World Health Organization (WHO) European Region, European Centre for Disease Prevention and Control (ECDC) and other partners have articulated potential solutions that are embedded into the new global approach for TB control, the End TB Strategy [2-5]. Within the context of the TB elimination framework for low-incidence countries in Western Europe [5], and the TB action plan for the WHO European Region 2016-2020 that addresses the whole region with greater emphasis on high-burden countries [6], it is apparent that concrete trans-national evidence-based interventional projects are needed. To address the high disease burden of TB in vulnerable EU populations we have formed the Early DETECTion of tuberculosis consortium (E-DETECT TB). It brings together world leading TB experts in national public health agencies with industry and major academic centres, and its membership reflects the incidence of $\mathrm{TB}$ in different EU countries (figure 1). E-DETECT TB uses evidenced-based approaches to target vulnerable populations, including migrants to the EU, homeless persons, prisoners, problem drug users and those with MDR-TB (table 1). Here we outline the key objectives and progress of this major European TB initiative with a special focus on migrant screening in Italy and active case finding in vulnerable groups in Romania.

Romania has the highest TB burden in the EU/EEA with more than a quarter (15195) of the reported patients, and with the highest notification rate of 76 per 100000 population, which is seven times higher than the EU/EEA average. The number of patients in Romania has declined dramatically since 2005 when 


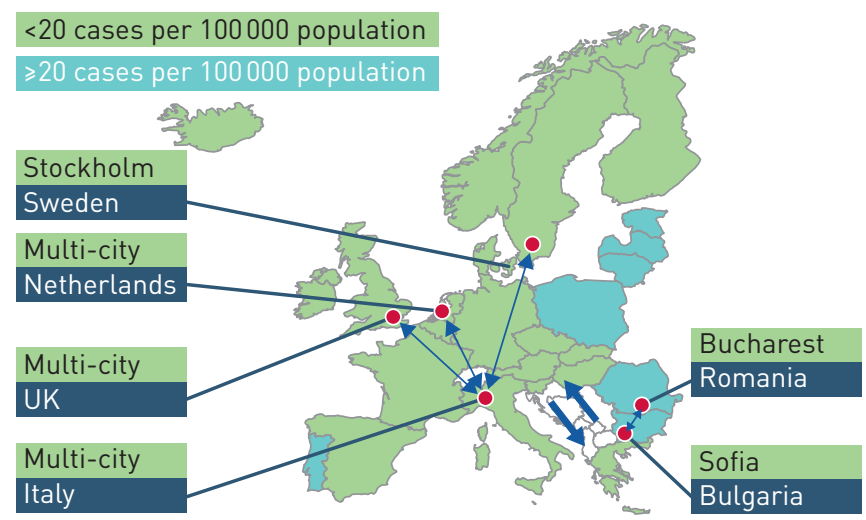

FIGURE 1 Map of Europe showing E-DETECT TB consortium partner countries and burden of tuberculosis.

almost 30000 patients were notified. The decline, however, is not observed among vulnerable populations, such as prisoners, homeless individuals, those with a history of drug use or in the Roma population. The E-DETECT TB project aims to apply innovative diagnostic tools, such as digital radiography with computer-automated reading and rapid bacteriological tests such as Xpert MTB/RIF, to improve early detection and diagnosis of TB in these vulnerable populations. The project also aims to strengthen care integration using an outreach strategy for the same vulnerable populations by providing a one-stop "shop" (clinic) which brings together all required procedures, social support for vulnerable groups, peer support, and close links to the national TB programme to ensure treatment completion. A pilot will be conducted in Bulgaria to assess and demonstrate the feasibility of the intervention. Bulgaria was selected due to the intermediate TB incidence and geographical proximity to Romania. This, together with the evaluation of effectiveness and cost-effectiveness, will inform policymakers on the potential for sustainability and scale-up in Romania and other Eastern European countries.

TB cases of foreign origin constitute about a third of all cases in the EU/EEA [1]. In low-incidence countries, trends in TB incidence are driven largely by international migration dynamics, and TB incidence rates among foreign-born individuals are usually several times higher than among non-foreign-born individuals. For example, in northern European countries such as Sweden and Norway over four in every five cases are of foreign origin. Consequently, tackling TB in migrants, especially migrant groups with additional vulnerabilities, is a priority [7-9]. Important vulnerable populations in Western Europe include asylum seekers and other migrants who arrive through routes that preclude pre-arrival TB screening. Most

TABLE 1 Summary table of E-DETECT TB interventions and outcomes

\begin{tabular}{ll} 
Work package & Interventions \\
\hline $\begin{array}{c}\text { Outreach activity in } \\
\text { Romania and Bulgaria }\end{array}$ & $\begin{array}{r}\text { Mobile digital radiography, } \\
\text { computer-aided diagnostics, mobile } \\
\text { provision of Xpert test, social support } \\
\text { and integration with hospital care }\end{array}$
\end{tabular}

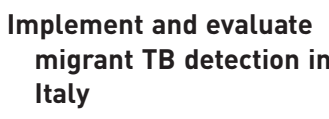

\section{Main outcome(s)}

Number of active TB cases detected using mobile radiography van in Romania and Bulgaria

Number of patients treated in Romania and Bulgaria

Latent TB testing in settled migrants (tuberculin skin test, interferon gamma release assayl

Active TB screening in newly arrived migrants (questionnaire, Xpert ULTRA)

Migrant TB screening database

Data from multiple countries

Survey of national programme managers Review of existing policies
Number of latent and active TB cases detected in migrants in Italy

Linkage to care among detected migrants in Italy

Multi-country data platforms for migrant TB established and analysed

Synthesis of best practice for developing national action plans

TB: tuberculosis. 
published evidence currently originates from the countries of resettlement of these migrants: there are reasons to predict that the yield of screening interventions, as well as the determinants of screening completion, will be different in countries of first arrival, such as Italy, Greece and Spain. The E-DETECT $\mathrm{TB}$ project aims at acquiring new insights with screening strategies for these vulnerable groups arriving in Italy. A two-pronged approach is adopted. Early detection of those arriving with symptoms is the main priority as an early diagnosis is instrumental to start the appropriate treatment and prevent transmission [10]. Under these conditions, screening for active disease using a mobile, fast, sensitive and specific molecular assay such the Xpert ULTRA assay is an interesting approach that warrants evaluation to determine its accuracy and cost-effectiveness. Recent data suggests that the Xpert ULTRA assay has an increased sensitivity compared with the previous versions of the Xpert MTB/RIF assay and retains the specificity [11]. A complementary approach is detection and treatment of latent TB infection, which represents the main source of TB cases in immigrants. While probing new testing tools (i.e. new versions of the interferon gamma release assays) we particularly focus on a healthcare delivery system that can maximise screening and treatment completion rates. Monitoring and evaluation of TB screening among migrants is another important part of the intervention. An electronic-health (eHealth) system called E-DETECT TB has been developed to address the need for a user-friendly, fast, mobile tool to register screening results among migrants directly at the point of arrival and at relocation sites. The E-DETECT TB system includes a smartphone application for mobile data recording, a java-based software and a synchronised electronic database, offering the opportunity to standardise screening practice and collect good-quality data. The E-DETECT TB system provides the opportunity to evaluate the public health use of this strategy in migrants and to investigate the potential for subsequent scale-up. However, the exchange of health information for individual migrants within a country and between countries remains a largely unresolved issue.

More broadly, the E-DETECT TB project assists the development of a robust regional monitoring and evaluation mechanism underpinned by the best quality operational data to support the identification of optimal approaches to diagnose latent and active TB and to ensure treatment completion. Many European TB programmes generate data on screening; however, the limited number of cases in each member state prevents the investigation of measures especially within subgroups. The E-DETECT TB project will provide a platform for the pooling of latent and active TB screening data from multiple countries to support future research into the best approaches for screening and evaluation of strategies to target risk groups. We have selected E-DETECT TB partner countries (Sweden, the Netherlands, Italy and the UK) as the initial countries to contribute data and have secured agreement to include additional EU/EEA countries in due course. This new data platform should complement existing ECDC surveillance systems and provide useful data to inform future policymaking in member states.

A robustly designed and well-implemented national TB strategy is regarded as key to successful national TB control [12]. One component of the E-DETECT TB project aims to collate the best evidence from published literature and national experts to inform a TB strategy prioritisation, action and support plan as a toolkit to aid national efforts to develop and implement national TB plans. To do this, we will collect evidence through two systematic reviews, a pan-European survey and an expert consultation exercise aimed at weighing the evidence and identifying specific barriers and facilitators to strategy implementation. In addition, we can also include relevant emerging evidence from other E-DETECT TB actions, including evidence on $\mathrm{TB}$ screening and tackling $\mathrm{TB}$ in vulnerable populations. After a pilot phase, we are hoping to make the resulting toolkit available to all EU/EEA member states.

In conclusion, all countries, even in the rich Europe, have a north and a south. National and international movement of populations has always existed, although the phenomenon has been increasingly visible over the past decade. This new media-driven attention needs to be properly oriented so as to ensure prevention of stigma and respect of human rights. Indeed, while some migrant groups are vulnerable populations, other vulnerable groups also need attention, e.g. homeless persons, Roma, drug addicts and prisoners, among others, including free-cost, rapid and quality diagnosis and treatment for TB, and for any other disease or comorbidity. The E-DETECT TB project has been designed to test novel outreach approaches supporting quality TB management in different countries and populations. By bringing together these multiple strands of activity, the consortium will play a key role in exploiting existing expertise among stakeholders in Europe, collaborating with the European Respiratory Society in its dissemination efforts, to maximise the impact of the intervention.

Although government commitment is a clear prerequisite to control and eliminate TB, and no elimination effort can work without the support of national TB programmes, the fight against the white plague is driven by multifactorial forces requiring multiple partners to join forces. National public health agencies, academia and the private sector including industry, civil society and professional organisations, in Europe the European Respiratory Society acts as a magnet for national associations, all need to collaborate closely to implement the End TB Strategy and to support and protect vulnerable populations. 
Acknowledgement: The authors gratefully acknowledge the E-DETECT TB (709624) project which has received funding from the European Union's Health Programme (2014-2020). The views expressed here are the authors only and are their sole responsibility; it cannot be considered to reflect the views of the European Commission and/or the Consumers, Health, Agriculture and Food Executive Agency or any other body of the EU. We are grateful to the staff of the consortium and project partners and colleagues who have commented on this manuscript (G. Stancanelli, V. Hack, M. Seng and D. Menezes).

Support statement: The E-DETECT TB project has received funding from the European Commission Consumers, Health, Agriculture and Food Executive Agency (grant number: 709624). Funding information for this article has been deposited with the Crossref Funder Registry.

Conflict of interest: I. Abubakar reports grants from European Commission to undertake the project reported in this manuscript.

\section{References}

1 European Centre for Disease Prevention and Control. Tuberculosis surveillance and monitoring in Europe, 2017. http://ecdc.europa.eu/en/publications-data/tuberculosis-surveillance-and-monitoring-europe-2017 Date last accessed October 17, 2017. Date last updated: March 20, 2017.

2 Broekmans JF, Migliori GB, Rieder HL, et al. European framework for tuberculosis control and elimination in countries with a low incidence. Recommendations of the World Health Organization (WHO), International Union Against Tuberculosis and Lung Disease (IUATLD) and Royal Netherlands Tuberculosis Association (KNCV) Working Group. Eur Respir J 2002; 19: 765-775.

3 D'Ambrosio L, Dara M, Tadolini M, et al. Tuberculosis elimination: theory and practice in Europe. Eur Respir J 2014; 43: 1410-1420.

4 European Centre for Disease Prevention and Control. Framework Action Plan to Fight Tuberculosis in the European Union. Solna, European Centre for Disease Prevention and Control, 2008.

5 Lönnroth K, Migliori GB, Abubakar I, et al. Towards tuberculosis elimination: an action framework for low-incidence countries. Eur Respir J 2015; 45: 928-952.

6 World Health Organization. Tuberculosis action plan for the WHO European Region 2016-2020. Copenhagen, WHO Regional Office for Europe, 2015.

7 Solovic I, Abubakar I, Sotgiu G, et al. Standard operating procedures for tuberculosis care. Eur Respir J 2017; 49: 1700515.

8 de Vries G, van Rest J, Meijer W, et al. Preventing and controlling tuberculosis among refugees in Europe: more needed for high-risk populations. Eur Respir J 2016; 48: 274-276.

9 Dara M, Solovic I, Goletti D, et al. Preventing and controlling tuberculosis among refugees in Europe: more is needed. Eur Respir J 2016; 48: 272-274.

10 Dara M, Solovic I, Sotgiu G, et al. Tuberculosis care among refugees arriving in Europe: a ERS/WHO Europe Region survey of current practices. Eur Respir J 2016; 48: 808-817.

11 Chakravorty S, Simmons AM, Rowneki M, et al. The new Xpert MTB/RIF Ultra: improving detection of Mycobacterium tuberculosis and resistance to rifampin in an assay suitable for point-of-care testing. mBio 2017; 8: e00812-17.

12 World Health Organization. Toolkit to develop a National Strategic Plan for TB prevention, care and control. Geneva, WHO, 2015. www.who.int/tb/publications/NSP_toolkit/en/ 